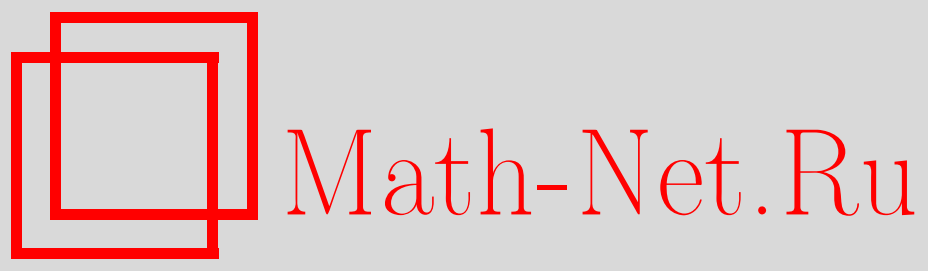

В. С. Рабинович, Устойчивость обратных операторов граничных задач в гладких расширяющихся областях, Функи. анализ и его прил., 2001, том 35, выпуск $4,85-87$

DOI: https://doi.org/10.4213/faa276

Использование Общероссийского математического портала MathNet.Ru подразумевает, что вы прочитали и согласны с пользовательским соглашением

http://www.mathnet.ru/rus/agreement

Параметры загрузки:

IP : 54.164 .48 .24

26 апреля 2023 г., 10:52:44

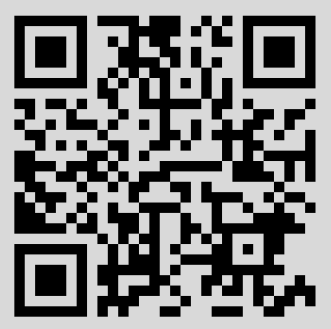


УДК 517.9

\title{
Устойчивость обратных операторов граничных задач в гладких расширяющихся областя ${ }^{*}$
}

\author{
(c) 2001. В. С. РАБинович
}

В работе рассматривается вопрос о равномерной ограниченности обратных операторов краевых задач в расширяющейся системе областей с гладкими границами при условии, что коэффициенты дифференциальных операторов краевой задачи принадлежат классу $C_{b}^{\infty}\left(\mathbb{R}^{n}\right)$ ограниченных на $\mathbb{R}^{n}$ вместе со всеми производными функций. Таким образом, никаких условий стабилизации на бесконечности на коэффициенты не налагается. (Класс $C_{b}^{\infty}\left(\mathbb{R}^{n}\right)$ содержит осциллирующие функции, например, равномерные почти периодические функции на $\mathbb{R}^{n}$.)

Устойчивость обратных операторов граничных задач является базой при исследовании связи между решениями граничных задач в ограниченных областях и во всем пространстве. Такая связь рассматривалась в монографии [1] при жестких условиях стабилизации коэффициентов дифференциальных операторов на бесконечности к константам и в работах $[2,3]$ при условии стабилизации коэффициентов к константам на бесконечности вдоль лучей в $\mathbb{R}^{n}$. Задачи с параметром в расширяющихся гладких областях рассматривались в $[4,5]$. Предлагаемый здесь подход к проблеме существенно отличается от подходов цитируемых работ. $\mathrm{K}$ указанной задаче впервые применяется метод предельных операторов, развитый автором для исследования фредгольмовости граничных задач в работах $[6,7]$.

1. Постановка задачи. Пусть $D$ есть ограниченная область в $\mathbb{R}^{n}$, содержащая начало координат и имеющая гладкую границу. Положим

$$
D_{r}=\left\{x \in \mathbb{R}^{n}: x / r \in D, r>0\right\} .
$$

Мы рассматриваем в области $D_{r}$ граничную задачу

где

$$
A u=f, \quad x \in D_{r}, \quad B_{1} u=f_{1}, \ldots, B_{m} u=f_{m}, \quad x \in \partial D_{r},
$$

$$
A=\sum_{|\alpha| \leqslant 2 m} a_{\alpha}(x) D^{\alpha}, \quad B_{j}=\sum_{|\alpha| \leqslant m_{j}} b_{\alpha j}(x) D^{\alpha}, \quad j=1, \ldots, m,
$$

- дифференциальные операторы с коэффициентами из пространства $C_{b}^{\infty}\left(\mathbb{R}^{n}\right)$. Сопоставим задаче (1) оператор

$$
\begin{aligned}
& \mathfrak{A}_{r}: X_{r}=H^{s}\left(D_{r}\right) \rightarrow Y_{r}=H^{s-2 m}\left(D_{r}\right) \bigoplus_{j=1}^{m} H^{s-m_{j}-1 / 2}\left(\partial D_{r}\right), \\
& \text { ax } m_{j}+1 / 2 .
\end{aligned}
$$

где $s>\max m_{j}+1 / 2$.

Мы рассматриваем вопрос об устойчивости семейства $\mathfrak{A}_{r}: X_{r} \rightarrow Y_{r}$ при больших $r$, т. е. вопрос о существовании $r_{0}>0$, такого, что операторы $\mathfrak{A}_{r}: X_{r} \rightarrow Y_{r}$ обратимы, если $r \geqslant r_{0}$, и

$$
\sup _{r \geqslant r_{0}}\left\|\mathfrak{A}_{r}^{-1}\right\|_{Y_{r} \rightarrow X_{r}}<\infty .
$$

2. Формулировка основных результатов. Для формулировки основных результатов работы мы нуждаемся в некоторых определениях. Пусть $\omega \neq 0$. Обозначим через $\eta_{\omega}$ бесконечно удаленную точку, отвечающую лучу $L_{\omega}$, выходя-

*Работа выполнена при поддержке проекта CONACYT 32424-E. 
щему из начала координат и проходящему через $\omega$. Мы определим окрестности бесконечно удаленной точки $\eta_{\omega}$ как множества

$$
U_{R, W}=\left\{x \in \mathbb{R}^{n}:|x|>R, x \in W\right\},
$$

где $W$ есть открытый конус с вершиной в начале координат, содержащий луч $L_{\omega}$. Будем говорить, что последовательность $\left\{h_{m}\right\}, h_{m} \in \mathbb{Z}^{n}$, сходится к $\eta_{\omega}$, если для каждой окрестности $U_{R, W}$ точки $\eta_{\omega}$ найдется $m_{0}$, такое, что $h_{m} \in U_{R, W}$, если $m \geqslant m_{0}$. Пусть $a(x) \in C_{b}^{\infty}\left(\mathbb{R}^{n}\right)$ и $\left\{h_{m}\right\}, h_{m} \in \mathbb{Z}^{n}$, сходится к $\eta_{\omega}$. Тогда найдется подпоследовательность $\left\{h_{m_{k}}\right\}$, сходящаяся к $\eta_{\omega}$, такая, что существует предел в $C^{\infty}$-топологии $\lim _{k \rightarrow \infty} a\left(x+h_{m_{k}}\right)=a^{h}(x)$. Нетрудно проверить, что $a_{h}(x) \in C_{b}^{\infty}\left(\mathbb{R}^{n}\right)$. Пусть последовательность $\left\{h_{m}\right\}$, сходящаяся к $\eta_{\omega}$, такова, что существуют пределы

$$
\lim _{m \rightarrow \infty} a_{\alpha}\left(x+h_{m}\right)=a_{\alpha}^{h}(x), \quad \lim _{m \rightarrow \infty} b_{\alpha j}\left(x+h_{m}\right)=b_{\alpha j}^{h}(x), \quad j=1, \ldots, m,
$$

в $C^{\infty}$-топологии. Обозначим через $A^{h}, B_{j}^{h}$ дифференциальные операторы с коэффициентами $a_{\alpha}^{h}(x)$ и $b_{\alpha j}^{h}(x)$ соответственно и назовем эти операторы предельными.

Пусть $v \in \partial D$. Обозначим через $\mathbf{H}_{v}$ полупространство, ограниченное касательным пространством к $\partial D$ в точке $v$ и содержащее внутреннюю нормаль к $\partial D$ в точке $v$. Положим $\mathbf{H}_{v}^{0}=\mathbf{H}_{v}-v$.

Теорема 1. Семейство $\mathfrak{A}_{r}: X_{r} \rightarrow Y_{r}$ устойчиво при больших $r$ тогда $u$ только тогда, когда

1) оператор $A: H^{s}\left(\mathbb{R}^{n}\right) \rightarrow H^{s-2 m}\left(\mathbb{R}^{n}\right)$ обратим;

2) для каждой точки $v \in \partial D$ и каждой последовательности $\left\{h_{m}\right\}$, сходящейся к $\eta_{v}$, такой, что предельнье операторьь $A^{h}, B_{j}^{h}, j=1, \ldots, m$, существуют, оператор граничной задачи в полупространстве

$$
\mathfrak{A}_{\mathbf{H}_{v}^{0}}^{h}: H^{s}\left(\mathbf{H}_{v}^{0}\right) \rightarrow H^{s-2 m}\left(\mathbf{H}_{v}^{0}\right) \bigoplus_{j=1}^{m} H^{s-m_{j}-1 / 2}\left(\partial \mathbf{H}_{v}^{0}\right)
$$

определяемьй внутренним оператором $A^{h}$ и граничными операторами $B_{j}^{h}$, $j=1, \ldots, m$, обратим и нормы обратных операторов $\left(\mathfrak{A}_{\mathbf{H}_{v}^{0}}^{h}\right)^{-1}$ равномерно ограничень.

Рассмотрим теперь следующую граничную задачу:

$$
A u_{r}=f_{r}, \quad x \in D_{r}, \quad B_{j} u_{r}=0, \quad x \in \partial D_{r}, j=1, \ldots, m,
$$

где $A$ и $B_{j}$ вводились раньше, а $f_{r}$ есть ограничение функции $f \in H^{s-2 m}\left(\mathbb{R}^{n}\right)$ на $D_{r}$. Теорема 1 имеет такое важное следствие:

Теорема 2. Пусть выполнены условия теоремы 1. Тогда существует $r_{0}>0$, такое, ито при $r \geqslant r_{0}$ задача (2) имеет единственное решение $u_{r}$. Более того,

$$
\lim _{r \rightarrow 0}\left\|\hat{u}_{r}-u_{r}\right\|_{H^{s}\left(D_{r}\right)}=0
$$

где $\hat{u}_{r}$ - ограничение на $D_{r}$ единственного решения $u \in H^{s}\left(\mathbb{R}^{n}\right)$ уравнения

$$
A u=f, \quad f \in H^{s-2 m}\left(\mathbb{R}^{n}\right) .
$$

ПримеР. Пусть $D(\ni 0)$ - область с гладкой границей $\partial D$. Рассмотрим задачу Дирихле для оператора Шрёдингера в области $D_{r}$

$$
(-\Delta+q(x)) u_{r}=f_{r}, \quad x \in D_{r},\left.u_{r}\right|_{\partial D_{r}}=\varphi_{r} .
$$


Обозначим через $\mathfrak{A}_{r}: H^{s}\left(D_{r}\right) \rightarrow H^{s-2}\left(D_{r}\right) \oplus H^{s-1 / 2}\left(\partial D_{r}\right), s>1 / 2$, оператор задачи (3). Предположим, что потенциал $q(x)$ медленно меняется на бесконечности, т. е. $q(x) \in C_{b}^{\infty}\left(\mathbb{R}^{n}\right)$ и $\lim _{x \rightarrow \infty} \partial_{x_{j}} q(x)=0, j=1, \ldots, n$. Можно показать, что в этом случае пределы $\lim _{k \rightarrow \infty} q\left(x+h_{k}\right), h_{k} \rightarrow \infty$ (если они существуют), не зависят от $x$. Кроме этого, предположим, что

$$
\inf _{\mathbb{R}^{n}} \operatorname{Im} q(x) \geqslant 0, \quad \lim _{r \rightarrow \infty} \inf _{|x|>r} \operatorname{Im} q(x)>0 .
$$

Применяя исчисление псевдодифференциальных операторов с медленно меняющимися символами [8], можно показать, что оператор $A=-\Delta+q(x): H^{s} \rightarrow H^{s-2}$ при условии (4) фредгольмов и имеет индекс нуль. Более того, из условия (4) и теоремы о единственности продолжения для решений эллиптических уравнений второго порядка (см., например, [9, с. 389]) получаем, что ядро оператора $A$ тривиально. Следовательно, $A$ обратим, т. е. условие 1) теоремы 1 выполнено.

Проверим условие 2) теоремы 1 . В нашем случае операторы $\mathfrak{A}_{\mathbf{H}_{v}^{h}}^{h}: H^{s}\left(\mathbf{H}_{v}^{0}\right) \rightarrow$ $H^{s-2}\left(\mathbf{H}_{v}^{0}\right) \oplus H^{s-1 / 2}\left(\partial \mathbf{H}_{v}^{0}\right)$ есть операторы задач Дирихле в полупространствах $\mathbf{H}_{v}^{0}$ для дифференциальных операторов $-\Delta+q_{h}$, где $q_{h}(\in \mathbb{C})-$ частичные пределы $q(x)$ при $x$, стремящемся к бесконечно удаленной точке $\eta_{v}$. Из условия (4) следует, что $\operatorname{Im} q_{h}>0$, и, используя теорию эллиптических операторов в полупространстве (см., например, [10]), получаем обратимость операторов $\mathfrak{A}_{\mathbf{H}_{v}^{0}}^{h}$. Более того, условие (10) влечет за собой равномерную ограниченность обратных операторов $\left(\mathfrak{A}_{\mathbf{H}_{v}^{0}}^{h}\right)^{-1}$.

Таким образом, все условия теоремы 1 выполнены, и, следовательно, семейство

устойчиво.

$$
\mathfrak{A}_{r}: H^{s}(D) \rightarrow H^{s-2}\left(D_{r}\right) \oplus H^{s-1 / 2}\left(\partial D_{r}\right), \quad s>1 / 2,
$$

Пусть $f \in H^{s-2}\left(\mathbf{R}^{n}\right), s>1 / 2$, и $f_{r}$ есть ограничение функции $f$ на $\mathscr{D}_{r}$, $\varphi_{r}=0$ для всех $r>0$. Тогда, как следует из теоремы 2 , существует $r_{0}$, такое, что для $r \geqslant r_{0}$ задача (3) имеет единственное решение $u_{r}$. Более того,

$$
\lim _{r \rightarrow 0}\left\|\hat{u}_{r}-u_{r}\right\|_{H^{s}\left(D_{r}\right)}=0,
$$

где $\hat{u}_{r}$ - ограничение на $D_{r}$ единственного решения $u \in H^{s}\left(\mathbb{R}^{n}\right)$ уравнения

$$
(-\Delta+q(x)) u=f, \quad f \in H^{s-2}\left(\mathbb{R}^{n}\right) .
$$

\section{ЛитЕРАТУРА}

1. Maz'ya V. G., Nazarov S. A., Plamenevskii B. A. Asymptotische Theorie elliptyischer Randwertaufgaben in singular gestrorten Gebeiten, V. 1. Akademie-Verlag, Berlin, 1991. 2. Докторский Л. Р. ДАН СССР, Сер. матем., 229, №6, 1303-1305 (1976). 3. Докторский Л. Р. Сиб. матем. ж., 20, №6, 1249-1260 (1979). 4. Шимон Л. Диф. уравнения, 9, № 8, 1482-1492 (1973). 5. Шимон Л. Матем. сб., 91, №4, 488-500 (1973). 6. Рабинович В. С. Труды С.-Петербург. матем. об-ва, 5, 349-379 (1998). 7. Рабинович В. С. ДАН СССР, сер. матем., 325, № 2, 237-241 (1992). 8. Грушин В. В. Функц. анализ и его прил., 4, вып. 3, 37-50 (1970). 9. Тейлор М. Псевдодифференциальные операторы. Мир, M., 1985. 10. Eskin G. I. Boundary Value Problems for Elliptic Pseudodifferential Equations. Transl. Math. Monogr., Amer. Math. Soc., Vol. 52, Providence, R.I., 1981.

Instituto Politecnco Nacional MEXICO

e-mail: rabinov@maya.esimez.ipn.mx

Поступило в редакцию

16 февраля 2000 г.

В переработанном виде 10 января 2001 г. 